\title{
Three dimensional sloshing of stratified liquid in a cylindrical tank
}

\author{
Wang Zhen ${ }^{\mathrm{a}, 1}$, Li Zou ${ }^{\mathrm{b}}$, Zhi Zong ${ }^{\mathrm{b}}$ \\ ${ }^{a}$ School of Mathematical Science, Dalian University of Technology, Dalian 116024, China \\ ${ }^{b}$ School of Naval Architecture, Dalian University of Technology, Dalian, 116085, China.
}

\begin{abstract}
The sloshing of stratified liquid with density varying with depth in a three dimensional cylindrical tank is considered in the framework of linearized theory. The flow of stratified liquid is no longer irrotional and the governing equation is no longer Laplacian. The stream function is also invalid for three dimensional sloshing, which is different from two dimensional sloshing. We adopt the governing equations and boundary conditions in terms of a pressure function and a density function to instead of velocity potential for uniform liquid. Separation of variables and Laplace transformation methods are used to solve the governing equations for the constant Brunt-Väisäl $\ddot{a}$ frequency. The Residue theorem is applied to calculate the inverse Laplace transformation and the resonant behavior is also analyzed. It is found that the natural frequencies are weakened by the stratification of the liquid density. New natural frequencies have appeared which do not exist for uniform liquid. We find that these new frequencies have some special characters; they make the motion history of free surface irregular, the amplitudes caused by new frequencies are remarkable, nearly two thirds of the amplitude caused by natural frequencies and all new frequencies are less than Brunt-Väisälä frequency $N$.
\end{abstract}

Keywords: continuous stratified liquid, resonance, sloshing, cylindrical tank

\section{Introduction}

Sloshing is a classic and important problem of fluid dynamics. It also has a wide range of applications in engineering, such as moving vehicle and structure containing a liquid with a free surface, ships, offshore platform, harbor reso5 nance. Resonant motion of the moving liquid can become extremely large if no or weak hydrodynamics damping is present. Sloshing is important for the safety of the structures $[1,2,3,3$, 4]. There are many publications devoted in sloshing

\footnotetext{
* Corresponding author

Email address: wangzhen@dlut.edu.cn (Wang Zhen)
}

Preprint submitted to Ocean Engineering

March 15, 2016

(C) 2016. This manuscript version is made available under the Elsevier user license http://www.elsevier.com/open-access/userlicense/1.0/ 
phenomenon, but most of them focus on the case of uniform density and two layer liquid [5, 6, 7, 8, 9, 10, 11].

Sloshing of liquid with variable density is also an important issue and applicable in engineering, such as waste storage tanks and crude oil storage tanks. Bandyopadhyay 12] indicated that a large number of high level waste (HLW) storage tanks at various U.S. Department of Energy (DOE) facilities contain liquid with nonuniform density. After prolonged storage, the waste material

15 and crude oil gradually deposit on the bottom of the tank. It may even form continuous variation of sludge at the tank base. So the difference of the density can be large from top to bottom of the tank. When gas is mixed into the liquid, the liquid can also be regarded as stratified liquid. In fact, there are also waves in the inner of the stratified liquid, which are called internal waves 13 .

Sloshing of continuously stratified liquid has its own characters and phenomenons. The predictions obtained through uniform density assumption may no longer hold. Thus, there is a need to understand the effect of nonuniform density on the dynamics response of the contained liquid. There are relatively less results for continuous stratified fluids. Most works assumed to be composed

25 of several layers with piecewise uniform densities [1, 6, 14]. This simplification introduces a pressure jump condition or shear stress at the interface of adjacent layers [1]. Exploratory studies on the dynamic response of tanks containing two liquids have been performed by Tang [6, 14]. Those studies show that the dynamic response of a tank containing two liquids is quite different from that of an 30 identical tank containing only one liquid. The sloshing wave height may increase significantly in a tank that contains two liquids. Therefore, it is necessary to understand the sloshing response of tanks that contain liquid with nonuniform density in order to design and evaluate the HLW storage tanks.

Many different numerical methods are developed for sloshing,such as 15, 16,

35 17, 18, 19]. The nonlinear resonance are also studied by numerical methods in Ref[20, 21]. The natural sloshing modes are very important for studying the sloshing phenomenon. There are also some works on sloshing modes and frequencies, such as 22, 23, 24, 25, 26]. An analytical solution for a liquid-filled tank with continuously varying density is a difficult task. Liquid with vari-

40 ational density continuously is no longer governed by irrotional and Laplacian type governing equation. For two dimensional rectangular tank, Wu[27] adopted stream function to analyze the sloshing, but stream function method does not work for the three dimensional sloshing. In this paper, we focus on the motion of the incompressible liquid with vertical stratified density in a three dimensional 45 cylindrical tank. We will deduce a differential equation in terms of pressure to represent the motion of stratified liquid with the help of the linearized momentum equations and the continuity equation. The governing equation in terms of pressure is different from that expressed by velocity potential when the density is constant. The boundary conditions are also expressed for the pressure, and

${ }_{50}$ then the Laplace transformation and separation of variables are applied to solve the problem.

The behavior of the motion of the stratified liquid is also different from that of uniform liquid. Motion of the stratified liquid leads to a more complicated 
equation for natural frequencies, and the dispersion relation is also more complicated. However it can be reduced to the result of constant density liquid.

\section{Governing equations and boundary conditions}

The motion of stratified liquid in a circular cylindrical tank with depth $d$ and radius $R_{0}$ is considered. Cylindrical coordinate system $(r, \theta, z)$ is adopted, the origin is located at the center of the static free surface and $z$ axis points upward which is the same in both coordinate systems.

The liquid is assumed to be inviscid. We also assume that the density depends on the depth of the fluid, in other words, the density $\rho_{0}(z)$ is a function of $z$. When the fluid is set into motion, we divide the density into static part $\rho_{0}(z)$ and dynamic part $\rho^{\prime}(x, y, z, t)$, i.e. $\rho(x, y, z, t)=\rho^{\prime}(x, y, z, t)+\rho_{0}(z)$. In what follows, we will denote $\rho_{0}(z), \rho(x, y, z, t)$ and $\rho^{\prime}(x, y, z, t)$ by $\rho_{0}, \rho$ and $\rho^{\prime}$, respectively. For still water, the velocities are set $\mathbf{v}=(u, v, w)=0$. The magnitude of static density $\rho_{0}$ is assumed as $O(1)$. The magnitudes of dynamics density $\rho^{\prime}$, velocities $\mathbf{v}=(u, v, w)$ and pressure function $p=p(x, y, z, t)$ for small motion are assumed as $O(\epsilon)$. Momentum equation can be expressed by

$$
\frac{d \mathbf{v}}{d t}=-\frac{1}{\rho} \operatorname{grad} p+\mathbf{F},
$$

where $\mathbf{F}=(0,0,-g)^{T}, g$ is the gravitational acceleration. Linearized momentum equation for small motion can be written as

$$
u_{t}=-\frac{1}{\rho} p_{x}, \quad v_{t}=-\frac{1}{\rho} p_{y}, \quad w_{t}=-\frac{1}{\rho} p_{z}-g .
$$

Since $\rho=\rho_{0}+\rho^{\prime}$, then the linearized momentum equation can be rewritten as

$$
\rho_{0} u_{t}=-p_{x}, \quad \rho_{0} v_{t}=-p_{y}, \quad \rho_{0} w_{t}=-p_{z}-\left(\rho^{\prime}+\rho_{0}\right) g .
$$

Here if $\rho$ is constant, then the liquid is irrotional and velocity potential function could be used to analyze sloshing of constant density liquid. But now $\rho_{0}$ is a function of $z$, then the rotation of velocity $\nabla \times \mathbf{v}$ is not always equal to zero. A different procedure is necessary.

Since sloshing of the liquid is considered in this paper, we can assume the liquid is incompressible as mentioned above, it means that

$$
\frac{d \rho}{d t}=\rho_{t}+u \rho_{x}+v \rho_{y}+w \rho_{z}=0,
$$

which can be found in many contexts, such as [4]. In the sense of linear approximation, it has a simple form by neglecting nonlinear terms

$$
\rho_{t}^{\prime}+\rho_{0 z} w=0 .
$$


Velocity component $w$ can be replaced by pressure $p$ with the aid of linearized momentum equation in $z$ component in Eq.(2), then the incompressible condition can be expressed by pressure and density as follows

$$
\rho_{t t}^{\prime}-\frac{\rho_{0 z}}{\rho_{0}} p_{z}-g \frac{\rho_{0 z}}{\rho_{0}}\left(\rho^{\prime}+\rho_{0}\right)=0 .
$$

${ }_{65}$ We note that Eq.(5) has the same form under the cylindrical coordinate.

Since the liquid is incompressible as shown in Eq.(3), the continuity equation

$$
\frac{d \rho}{d t}+\rho\left(u_{x}+v_{y}+w_{z}\right)=0
$$

takes the form as

$$
u_{x}+v_{y}+w_{z}=0 .
$$

Multiply Eq.(6) with $\rho_{0}$ and differentiate Eq.(6) with respect to $t$, we get an equation in terms of pressure $p$ and dynamic density $\rho^{\prime}$ by the aid of Eq.(2)

$$
\nabla^{2} p+\rho_{z}^{\prime} g-\frac{\rho_{0 z}}{\rho_{0}}\left(p_{z}+\rho^{\prime} g\right)=0,
$$

which also can be rewritten as

$$
p_{z z}+\frac{1}{r}\left(r p_{r}\right)_{r}+\frac{1}{r^{2}} p_{\theta \theta}+\rho_{z}^{\prime} g-\frac{\rho_{0 z}}{\rho_{0}}\left(p_{z}+\rho^{\prime} g\right)=0 .
$$

in cylindrical coordinates $(r, \theta, z)$.

Eq.(8) and Eq.(5) are the governing equations in the whole zone of the liquid under cylindrical coordinate. These equations are in terms of pressure function and density function that deduced from the momentum equation Eq.(2), continuity equation Eq.(6) and incompressible condition Eq.(3).

The dynamics boundary condition on the free surface $z=\eta(x, y, t)$ can be written as

$$
p(x, y, z=\eta, t)=0 .
$$

We will deduce a condition for pressure at mean level $z=0$. In order to match the governing equations which are in terms of pressure and density for varying density flow, linear approximation of the pressure at the mean surface level $z=0$ gives

$$
p(x, y, 0, t)+\frac{\partial p(x, y, 0, t)}{\partial z} \eta=0,
$$

where $\frac{\partial p(x, y, 0, t)}{\partial z}$ can be given by $-\rho_{0}(0) g$ with the aid of the third momentum equation of Eq.(2), which also called hydrostatic condition 3]. So Eq.(10) can be written as

$$
p(x, y, 0, t)-\rho_{0}(0) g \eta=0,
$$


This relation also gives a direct link between pressure and free surface. The expression of free surface will be given by the solution of pressure in next section.

Differentiating Eq. (11) with respect to $t$ twice, and considering the relation $\eta_{t}=w$ and linear momentum equation on the free surface $z=0$, we get

$$
p_{t t}+g p_{z}+g^{2}\left(\rho^{\prime}+\rho_{0}(0)\right)=0 \text { on } z=0 .
$$

At the bottom of the tank, the normal velocity should satisfy

$$
w(x, y, z=-d, t)=0
$$

which implies

$$
p_{z}+g\left[\rho^{\prime}+\rho_{0}(-d)\right]=0 \text { at } z=-d
$$

75 with the aid of the third momentum equation of Eq.(2) .

On the vertical cylindrical wall, the velocity of the fluid particles should be coined with the motion of solid wall $U(t)$ horizontally, in other word $u \vec{i}+v \vec{j}=$ $U \vec{i}+V \vec{j}$, Where $U$ and $V$ are the forced motions of the tank in directions $x$ and $y$ respectively. Let us consider the velocity in the direction of radius and express it by pressure through the momentum equation

$$
-\left.\frac{\partial p}{\partial r}\right|_{r=R_{0}}=\rho_{0} U_{t} \sin (\theta) \text {. }
$$

Equation system (5), (8), (12), (14) and (15) are the governing equations and boundary conditions with respect to pressure $p$ and density $\rho^{\prime}$. If we set $p_{1}=$

$p+g \int_{0}^{z} \rho_{0}(s) d s$, then the governing equations and boundary conditions can be converted into

$$
\begin{aligned}
& p_{1 z z}+\frac{1}{r}\left(r p_{1 r}\right)_{r}+\frac{1}{r^{2}} p_{1 \theta \theta}+\rho_{z}^{\prime} g+\frac{N^{2}}{g}\left(p_{1 z}+\rho^{\prime} g\right)=0,-d<z<0 \\
& \rho_{t t}^{\prime}+\frac{N^{2}}{g} p_{1 z}+N^{2} \rho^{\prime}=0,-d<z<0 \\
& p_{1 t t}+g p_{1 z}+g^{2} \rho^{\prime}=0, \text { on } z=0 \\
& p_{1 z}+g \rho^{\prime}=0, \text { on } z=-d, \\
& -\left.\frac{\partial p_{1}}{\partial r}\right|_{r=R_{0}}=\rho_{0} U_{t} \sin (\theta)
\end{aligned}
$$

where $N^{2}=-g \frac{\rho_{0 z}}{\rho_{0}}$ is the Brunt-Väisälä frequency. It represents a sort of maximum frequency for oscillations under gravity. We assume $N^{2}>0$ is constant in this paper to make sure the stratified liquid is stable, then the density distribution can be determined as $\rho_{0}=\rho_{0}(0) e^{-N^{2} z / g}$.

\section{Sloshing in the forced horizontal motion}

We denote $\bar{p}, \bar{\rho}^{\prime}$ and $\bar{U}$ as the Laplace transformation of $p, \rho^{\prime}$ and $U$ with respect to time $t$. We also assume the fluid is static at the starting point. The 
governing equation and boundary conditions could be transformed into

$$
\begin{aligned}
& \bar{p}_{1 z z}+\frac{1}{r}\left(r \bar{p}_{1 r}\right)_{r}+\frac{1}{r^{2}} \bar{p}_{1 \theta \theta}+\bar{\rho}_{z}^{\prime} g+\frac{N^{2}}{g}\left(\bar{p}_{1 z}+\bar{\rho}^{\prime} g\right)=0, \quad-d<z<0 \\
& s^{2} \bar{\rho}^{\prime}+\frac{N^{2}}{g} \bar{p}_{1 z}+N^{2} \bar{\rho}^{\prime}=0, \quad-d<z<0 \\
& s^{2} \bar{p}_{1}+g \bar{p}_{1 z}+g^{2} \bar{\rho}^{\prime}=0, \text { on } z=0 \\
& \bar{p}_{1 z}+g \bar{\rho}^{\prime}=0, \text { on } z=-d \\
& -\left.\frac{\partial \bar{p}_{1}}{\partial r}\right|_{r=R_{0}}=s \rho_{0} \bar{U} \sin (\theta), \quad \text { on } \quad r=R_{0} .
\end{aligned}
$$

From Eq.(22), we can obtain an explicit simple relation between $\overline{\rho^{\prime}}$ and $\overline{p_{1}}$,

$$
\overline{\rho^{\prime}}=-\frac{N^{2} \bar{p}_{1 z} / g}{s^{2}+N^{2}} .
$$

Then Eq.(21) can be rewritten in terms of pressure

$$
\frac{s^{2}}{s^{2}+N^{2}} \bar{p}_{1 z z}+\frac{1}{r}\left(r \bar{p}_{1 r}\right)_{r}+\frac{1}{r^{2}} \bar{p}_{1 \theta \theta}+\frac{N^{2}}{g} \frac{s^{2}}{s^{2}+N^{2}} \bar{p}_{1 z}=0,-d<z<0,
$$

and free surface and bottom boundary conditions are transformed into

$$
\begin{array}{r}
\bar{p}_{1}+\frac{g}{s^{2}+N^{2}} \bar{p}_{1 z}=0, \text { on } z=0 \\
\bar{p}_{1 z}=0, \text { on } z=-d .
\end{array}
$$

The pressure function $\bar{p}_{1}$ should have a standard separated variables form, since it satisfies Eq.(21). Considered the boundary conditions on the solid walls of forced motion Eq.(25), pressure function $\bar{p}_{1}$ can be expanded as

$$
\begin{aligned}
\bar{p}_{1}= & -s \rho_{0}(z) \bar{U} r \sin (\theta)+\sum_{j, n=1}^{\infty} \bar{f}_{n, j}(z, s) J_{n}\left(\mu_{n, j} \frac{r}{R_{0}}\right) \sin (n \theta) \\
& +\sum_{j, n=1}^{\infty} \bar{g}_{n, j}(z, s) J_{n}\left(\mu_{n, j} \frac{r}{R_{0}}\right) \cos (n \theta),
\end{aligned}
$$

where $\bar{f}_{n, j}(z, s)$ and $\bar{g}_{n, j}(z, s)$ are the Laplace transformations of $f_{n, j}(z, t)$ and $g_{n, j}(z, t), J_{n}\left(\mu_{n, j} \frac{r}{R_{0}}\right)$ is the $\mathrm{n}$-th order Bessel function and $\mu_{n, j}$ is the $\mathrm{j}$-th zero point of the derivative of $J_{n}$, i.e. $J_{n}^{\prime}\left(\mu_{n, j}\right)=0$.

In what follows, we will determine $\bar{f}_{n, j}(z, s)$ and $\bar{g}_{n, j}(z, s)$ by satisfying the governing equation Eq. (27) and boundary conditions Eqs.(28) (29). Substituting the expression of $\bar{p}_{1}$ into the governing equation Eq.(27) and using the orthogonality of the sine functions on interval $[0,2 \pi]$ and Bessel functions on distribution $\left[0, R_{0}\right]$, we get a series of equations in terms of $f_{n, j}(z, s)$ and $g_{n, j}(z, s)$. From these equations, we can conclude that functions $f_{n, j}(z, s)$ for $n \geq 2, j \geq 1$ 
and $g_{n, j}(z, s)$ for $n \geq 1, j \geq 1$ vanish, only $f_{1, j}(z, s)$ is nonzero function. In fact, the detailed governing equations are as follows

$$
\begin{aligned}
& \frac{\partial^{2} \bar{f}_{n, j}}{\partial z^{2}}-\left(\frac{\mu_{n, j}}{R_{0}}\right)^{2}\left(1+\frac{N^{2}}{s^{2}}\right) \bar{f}_{n, j}+\frac{N^{2}}{g} \frac{\partial \bar{f}_{n, j}}{\partial z}=0, \quad n \geq 2, j \geq 1 \\
& \frac{\partial^{2} \bar{g}_{n, j}}{\partial z^{2}}-\left(\frac{\mu_{n, j}}{R_{0}}\right)^{2}\left(1+\frac{N^{2}}{s^{2}}\right) \bar{g}_{n, j}+\frac{N^{2}}{g} \frac{\partial \bar{g}_{n, j}}{\partial z}=0, \quad n \geq 1, j \geq 1 .
\end{aligned}
$$

The dynamic boundary condition gives

$$
\begin{aligned}
& \bar{f}_{n, j}+\frac{g}{s^{2}+N^{2}} \frac{\partial \bar{f}_{n, j}}{\partial z}=0, \quad n \geq 2, j \geq 1 \\
& \bar{g}_{n, j}+\frac{g}{s^{2}+N^{2}} \frac{\partial \bar{g}_{n, j}}{\partial z}=0, \quad n \geq 1, j \geq 1
\end{aligned}
$$

on surface $z=0$ and the bottom boundary condition gives

$$
\begin{aligned}
& \frac{\partial \bar{f}_{n, j}}{\partial z}=0, \quad n \geq 2, j \geq 1 \\
& \frac{\partial \bar{g}_{n, j}}{\partial z}=0, \quad n \geq 1, j \geq 1
\end{aligned}
$$

on $z=-d$.

The solutions of above equation system are all zero, i.e. $\bar{f}_{n, j}(z, s)=0$, for $n \geq 2, j \geq 1$ and $\bar{g}_{n, j}(z, s)=0$ for $n \geq 1, j \geq 1$. Then the expression of the pressure $\bar{p}_{1}$ only depends on $\bar{f}_{1, j}(z, s)$. Substituting the expression of $\bar{p}_{1}$ into Eq.(27) and using the orthogonality of sine function and Bessel function give

$$
\frac{\partial^{2} \bar{f}_{1, j}}{\partial z^{2}}-\left(\frac{\mu_{1, j}}{R_{0}}\right)^{2}\left(1+\frac{N^{2}}{s^{2}}\right) \bar{f}_{1, j}+\frac{N^{2}}{g} \frac{\partial \bar{f}_{1, j}}{\partial z}=s\left(\rho_{0 z z}+\frac{N^{2}}{g} \rho_{0 z}\right) \bar{U} C_{1, j},
$$

where

$$
C_{1, j}=\frac{\int_{0}^{R_{0}} r^{2} J_{1}\left(\mu_{1, j} \frac{r}{R_{0}}\right) d r}{\int_{0}^{R_{0}} r J_{1}^{2}\left(\mu_{1, j} \frac{r}{R_{0}}\right) d r}
$$

Here it is noted that the right hand side of above equation equals to zero. Since $N^{2}=-g \frac{\rho_{0 z}}{\rho_{0}}$ is assumed as constant, then expression $\rho_{0 z z}+N^{2} \rho_{0 z} / g$ can be written as $\rho_{0}\left(\frac{\rho_{0 z}}{\rho_{0}}\right)$, so it equals to zero.

The solution of Eq. (37) can be given by

$$
\bar{f}_{1, j}=A_{1, j} e^{\alpha_{1, j} z}+B_{1, j} e^{\beta_{1, j} z}
$$

where

$$
\begin{aligned}
& \alpha_{1, j}=-\frac{N^{2}}{2 g}+\sqrt{N^{4} /\left(4 g^{2}\right)+\left(1+N^{2} / s^{2}\right) \mu_{1, j}^{2} / R_{0}^{2}}, \\
& \beta_{1, j}=-\frac{N^{2}}{2 g}-\sqrt{N^{4} /\left(4 g^{2}\right)+\left(1+N^{2} / s^{2}\right) \mu_{1, j}^{2} / R_{0}^{2}},
\end{aligned}
$$


and $A_{1, j}$ and $B_{1, j}$ will be determined by the boundary conditions Eq.(28) and Eq.(29). Substituting Eq.(30) into Eq.(28) and using the orthogonality of sine functions and Bessel functions, the free surface boundary condition can be written as

$$
\bar{f}_{1, j}+\frac{g}{s^{2}+N^{2}} \frac{\partial \bar{f}_{1, j}}{\partial z}=\frac{s^{3} \rho_{0}(0) \bar{U}}{s^{2}+N^{2}} C_{1, j}, j \geq 1
$$

on free surface $z=0$. Substituting Eq.(30) into Eq.(29) and using the orthogonality of sine functions and Bessel functions, the bottom boundary condition can be written as

$$
\frac{\partial \bar{f}_{1, j}}{\partial z}=s \rho_{0 z}(-d) \bar{U} C_{1, j}, j \geq 1
$$

at $z=-d$. Using Eq.(38) and Eq.(41), we can get

$$
\left(s^{2}+N^{2}+g \alpha_{1, j}\right) A_{1, j}+\left(s^{2}+N^{2}+g \beta_{1, j}\right) B_{1, j}=s^{3} \rho_{0}(0) \bar{U} C_{1, j} .
$$

At the bottom of tank, Eq.(38) and Eq.(42) give

$$
\alpha_{1, j} A_{1, j} e^{-\alpha_{1, j} d}+\beta_{1, j} B_{1, j} e^{-\beta_{1, j} d}=s \rho_{0 z}(-d) \bar{U} C_{1, j} .
$$

Equations Eq.(43) and Eq.(44) admit $A_{1, j}$ and $B_{1, j}$ in the form of

$$
\begin{aligned}
A_{1, j} & =\frac{\beta_{1, j} e^{-\beta_{1, j} d} s^{3} \rho_{0}(0)-s \rho_{0 z}(-d)\left(s^{2}+N^{2}+g \beta_{1, j}\right)}{\left(s^{2}+N^{2}+g \alpha_{1, j}\right) \beta_{1, j} e^{-\beta_{1, j} d}-\left(s^{2}+N^{2}+g \beta_{1, j}\right) \alpha_{1, j} e^{-\alpha_{1, j} d}} \bar{U} C_{1, j}, \\
B_{1, j} & =\frac{s \rho_{0 z}(-d)\left(s^{2}+N^{2}+g \alpha_{1, j}\right)-\alpha_{1, j} e^{-\alpha_{1, j} d} s^{3} \rho_{0}(0)}{\left(s^{2}+N^{2}+g \alpha_{1, j}\right) \beta_{1, j} e^{-\beta_{1, j} d}-\left(s^{2}+N^{2}+g \beta_{1, j}\right) \alpha_{1, j} e^{-\alpha_{1, j} d}} \bar{U} C_{1, j} .
\end{aligned}
$$

After some simple calculations and simplifications, then $\bar{f}_{1, j}$ can be given as

$$
\bar{f}_{1, j}=\frac{s D_{j}(z, s)}{E_{j}(s)} \frac{s^{2}}{s^{2}+N^{2}} \bar{U} C_{1, j},
$$

where

$$
\begin{aligned}
& D_{j}(z, s)=\left(\beta_{1, j} e^{-\beta_{1, j} d+\alpha_{1, j} z}-\alpha_{1, j} e^{-\alpha_{1, j} d+\beta_{1, j} z}\right) \rho_{0}(0) s^{2} \\
& +\left[\left(s^{2}+N^{2}+g \alpha_{1, j}\right) e^{\beta_{1, j} z}-\left(s^{2}+N^{2}+g \beta_{1, j}\right) e^{\alpha_{1, j} z}\right] \rho_{0 z}(-d) \\
& E_{j}(s)=s^{2}\left(\beta_{1, j} e^{-\beta_{1, j} d}-\alpha_{1, j} e^{-\alpha_{1, j} d}\right)-g \mu_{1, j}^{2} / R_{0}^{2}\left(e^{-\beta_{1, j} d}-e^{-\alpha_{1, j} d}\right) .
\end{aligned}
$$

Now the detailed form of Laplace transformation of pressure $\overline{p_{1}}$ as given in Eq. (30) are determined, the pressure can be given by inverse Laplace transformation

$$
p_{1}=\frac{1}{2 \pi i} \int_{\Gamma} \bar{p}_{1} e^{s t} d s
$$


and the surface elevation is given by equation (13) in the sense of first approximation

$$
\eta(r, \theta, t)=\frac{p(r, \theta, 0, t)}{\rho_{0}(0) g},
$$

where $p(x, y, 0, t)$ can be given by Eq.(30), Eq.(38), Eq.(45) and Eq.(46). Finally the expression of free surface can be given as

$$
\begin{aligned}
& \eta(r, \theta, t)=\frac{p_{1}(r, \theta, 0, t)}{\rho_{0}(0) g} \\
& =-\frac{U_{t} r \sin (\theta)}{g}+\sum_{j=1}^{\infty} \frac{C_{1, j}}{\rho_{0}(0) g} J_{1}\left(\mu_{1, j} \frac{r}{R_{0}}\right) \sin (\theta) \frac{1}{2 \pi i} \int_{\Gamma} \frac{s F_{j}(s)}{E_{j}(s)\left(s^{2}+N^{2}\right)} \bar{U} e^{s t} d s
\end{aligned}
$$

where

$$
F_{j}(s)=\left(\alpha_{1, j}-\beta_{1, j}\right) g \rho_{0 z}(-d) s^{2}+\left(\beta_{1, j} e^{-\beta_{1, j} d}-\alpha_{1, j} e^{-\alpha_{1, j} d}\right) \rho_{0}(0) s^{4} .
$$

This result is deduced from Eqs.(16)-(20), which do not consider the effect of static water pressure. In fact, the static water pressure give no effect on surface

90 elevation. It is also can be deduced from relation $p_{1}=p+\int_{0}^{z} \rho_{0}(z) d z$, since static water pressure $\int_{0}^{z} \rho_{0}(z) d z$ vanishes on the free surface $z=0$.

\section{Results for forced sloshing}

Now we consider that the tank is in forced oscillation motion

$$
U(t)=U_{0}(1-\cos (\omega t))
$$

Its Laplace transformation is

$$
\bar{U}=\frac{U_{0} \omega^{2}}{s\left(s^{2}+\omega^{2}\right)},
$$

then the free surface can be given as

$$
\begin{aligned}
& \eta(r, \theta, t)=-\frac{U_{t} r \sin (\theta)}{g} \\
& +\sum_{j=1}^{\infty} \frac{C_{1, j}}{\rho_{0}(0) g} J_{1}\left(\mu_{1, j} \frac{r}{R_{0}}\right) \sin (\theta) \frac{1}{2 \pi i} \int_{\Gamma} \frac{F_{j}(s)}{E_{j}(s)} \frac{1}{s^{2}+N^{2}} \frac{U_{0} \omega^{2}}{s^{2}+\omega^{2}} e^{s t} d s,
\end{aligned}
$$

where $F_{j}(s)$ and $E_{j}(s)$ are given in Eq.(54) and Eq.(49) respectively.

In order to work out the inverse Laplace transformation for free surface Eq.(57), we should analyze the poles of the integrand. First, $\pm i \omega$ are the simple 
poles of the integrand; Secondly, the zero points of $E_{1, j}$ are also the poles of the integrand. Zero points of $E_{1, j}$ are equivalent to the roots of

$$
s^{2}+\frac{2 g^{2} \mu_{1, j}^{2} \tanh \left(\gamma_{j} d\right)}{N^{2} R_{0}^{2} \tanh \left(\gamma_{j} d\right)+2 g \gamma_{j} R_{0}^{2}}=0 .
$$

where

$$
\gamma_{j}=\frac{\alpha_{1, j}-\beta_{1, j}}{2}=\sqrt{\frac{N^{4}}{4 g^{2}}+\left(1+\frac{N^{2}}{s^{2}}\right) \frac{\mu_{1, j}^{2}}{R_{0}^{2}}} .
$$

Because $\gamma_{j}$ and $d$ are positive, so $\tanh \left(\gamma_{j} d\right)>0$, which means that $E_{1, j}(s)$ is 95 $s$ in $E_{1, j}(s)$ appears always in the form of $s^{2}$, then each imaginary solutions appeared always as a pair with its conjugate. So we can assume the roots of $E_{1, j}(s)$ are in the form of $s= \pm i \omega_{j, n}$, where $\omega_{j, n}$ is real. Furthermore, we can assume $\omega_{j, n}>0$, as if the positive number is solution then the opposite one is also a solution.

We use $\Delta_{j, n}$ to denote

$$
\Delta_{j, n}=\frac{N^{4}}{4 g^{2}}+\left(1-\frac{N^{2}}{\omega_{j, n}^{2}}\right) \frac{\mu_{1, j}^{2}}{R_{0}^{2}} .
$$

When $\Delta_{1, j} \geq 0$, in other words, we consider the case of

$$
\omega_{j, n} \geq \frac{\mu_{1, j} N}{\sqrt{N^{4} R_{0}^{2} /\left(4 g^{2}\right)+\mu_{1, j}^{2}}},
$$

then we find that the real roots should satisfy

$$
-\omega_{j, n}^{2}+\frac{2 g^{2} \mu_{1, j}^{2} \tanh \left[d \sqrt{\Delta_{j, n}}\right]}{N^{2} R_{0}^{2} \tanh \left[d \sqrt{\Delta_{j, n}}\right]+2 g R_{0}^{2} \sqrt{\Delta_{j, n}}}=0 .
$$

According to the monotonicity of the functions, we conclude that above equation has only one real solution. Figure 1 shows the effects of $N$ on the natural frequency $\omega_{1,1}$. The natural frequency decreases weakly as the parameter $N$ increases. The natural frequency of stratified liquid is smaller than the frequency of uniform liquid.

When $\Delta_{j, n}<0$, in other words, we consider the solution which satisfies

$$
0<\omega_{j, n}<\frac{\mu_{1, j} N}{\sqrt{N^{4} R_{0}^{2} /\left(4 g^{2}\right)+\mu_{1, j}^{2}}},
$$

then the right hand side of Eq. (58) can be rewritten as

$$
-\omega_{j, n}^{2}+\frac{2 g^{2} \mu_{1, j}^{2} \tan \left[d \sqrt{-\Delta_{j, n}}\right]}{N^{2} R_{0}^{2} \tan \left[d \sqrt{-\Delta_{j, n}}\right]+2 g R_{0}^{2} \sqrt{-\Delta_{j, n}}}=0,
$$




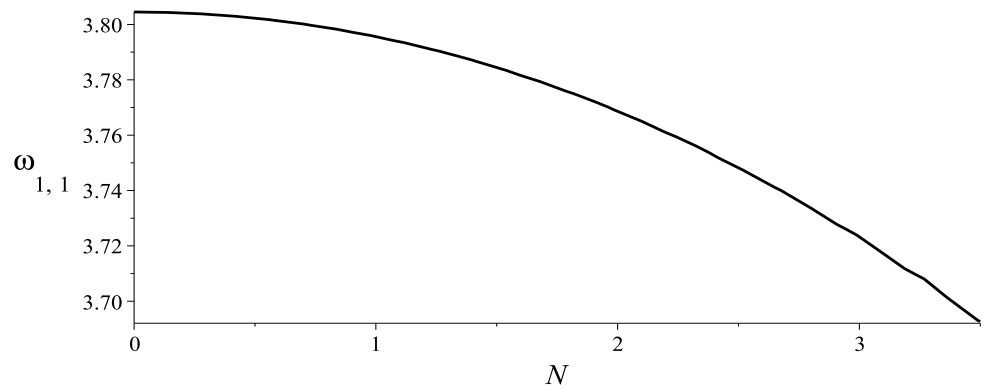

Figure 1: The effect of $N$ on the first natural frequency $\omega_{1,1}$ which is solved from Eq. 559) with $d=0.6, U_{0}=0.5, \rho_{0}(0)=1$ and $R_{0}=1$.

which admits infinite solutions.

Because the periodic function appears in the equation, the Eq. (600) may permit solution in range

$$
\begin{gathered}
\frac{\mu_{1, j} N}{\sqrt{N^{4} R_{0}^{2} /\left(4 g^{2}\right)+\mu_{1, j}^{2}+(n+1 / 2)^{2} \pi^{2} R_{0}^{2} / d^{2}}} \\
\leq \omega_{j, n+1} \leq \frac{\mu_{1, j} N}{\sqrt{N^{4} R_{0}^{2} /\left(4 g^{2}\right)+\mu_{1, j}^{2}+n^{2} \pi^{2} R_{0}^{2} / d^{2}}}
\end{gathered}
$$

for $n=1,2,3, \cdots$. We find that $\omega_{1,2}=0.25 \Omega_{1}$ is natural frequency approximately in interval Eq. (61) for $j=1, n=1$ by numerical method; $\omega_{1,3}=0.13 \Omega_{1}$ is also natural frequency in interval Eq.(61) for $j=1, n=2$ for $N=3$, where $\Omega_{1}$ is the natural frequency for uniform liquid sloshing. Eq.(61) represents infinite intervals as $n$ increasing. So there are infinite natural frequencies. This is different from the liquid with constant density.

The new natural frequencies tend to zero rapidly as $n$ increases for the fixed $j$. The solutions $\omega_{j, n}$ for large $n$ is too small to have practical significance. While the root of derivative of Bessel function $\mu_{1, j}$ increases along with $j$ for fixed $n$, the $j$-th solution of Eq. (60) $\omega_{j, n}$, which located in the interval Eq.(61), also increases along index $j$. On the other hand, all new natural frequencies are less than the Brunt-Väisälä frequency $N$, which can be deduced from the expression of

$$
0<\omega_{j, n}<\frac{\mu_{1, j} N}{\sqrt{N^{4} R_{0}^{2} /\left(4 g^{2}\right)+\mu_{1, j}^{2}}}<N
$$

for $n>1$. In fact, the zero points of the derivative of Bessel function has an approximate property

$$
\frac{(j-1 / 2) \pi}{\mu_{1, j}} \rightarrow 1, \text { as } j \rightarrow \infty .
$$

So the new natural frequencies $\omega_{j, n}$ tend to $N$ from left for fixed $n$. 
When $E_{j}(s)$ has only simple zeros $\pm i \omega_{j, n}$, which means $E_{j}\left( \pm i \omega_{j, n}\right)=0$ and $E_{j}^{\prime}\left( \pm i \omega_{j, n}\right) \neq 0$, then the inverse Laplace transformation of complex integration can be calculated by Residue theorem

$$
\begin{aligned}
& \frac{1}{2 \pi i} \int_{\Gamma} \frac{F_{j}(s)}{E_{j}(s)} \frac{1}{s^{2}+N^{2}} \frac{U_{0} \omega^{2}}{s^{2}+\omega^{2}} e^{s t} d s \\
& =\frac{U_{0} \omega}{N^{2}-\omega^{2}} \frac{F_{j}(i \omega)}{E_{j}(i \omega)} \sin (\omega t)+\sum_{n} \frac{F_{j}\left(i \omega_{j, n}\right)}{E_{j}^{\prime}\left(i \omega_{j, n}\right)} \frac{2 i}{N^{2}-\omega_{j, n}^{2}} \frac{U_{0} \omega^{2}}{\omega^{2}-\omega_{j, n}^{2}} \sin \left(\omega_{j, n} t\right) .
\end{aligned}
$$

The free surface can be given by

$$
\begin{aligned}
& \eta(r, \theta, t)=-\frac{U_{0} \sin (\omega t) \sin (\theta) r}{g}+\sum_{j=1}^{\infty} \frac{C_{1, j} U_{0} \omega^{2}}{\rho_{0}(0) g} J_{1}\left(\mu_{1, j} \frac{r}{R_{0}}\right) \sin (\theta) \times \\
& \left(\frac{F_{j}(i \omega)}{E_{j}(i \omega)\left(N^{2}-\omega^{2}\right) \omega} \sin (\omega t)+2 i \sum_{n} \frac{F_{j}\left(i \omega_{j, n}\right)}{E_{j}^{\prime}\left(i \omega_{j, n}\right)\left(N^{2}-\omega_{j, n}^{2}\right)} \frac{1}{\omega^{2}-\omega_{j, n}^{2}} \sin \left(\omega_{j, n} t\right)\right) .
\end{aligned}
$$

When $N=0$, i.e the density in the tank is constant, parameters mentioned above degenerate into

$$
\begin{aligned}
& \alpha_{1, j}=\frac{\mu_{1, j}}{R_{0}}, \quad \beta_{1, j}=-\frac{\mu_{1, j}}{R_{0}}, \quad \gamma_{1, j}=2 \frac{\mu_{1, j}}{R_{0}}, \\
& E_{j}(s)=-\frac{\mu_{1, j}}{R_{0}} s^{2}\left(e^{\frac{\mu_{1, j}}{R_{0}}}+e^{-\frac{\mu_{1, j}}{R_{0}}}\right)-g \frac{\mu_{1, j}^{2}}{R_{0}^{2}}\left(e^{\frac{\mu_{1, j}}{R_{0}}}-e^{-\frac{\mu_{1, j}}{R_{0}}}\right) .
\end{aligned}
$$

The expression of $E_{j}(s)$ implies it has a solution

$$
\omega_{j}^{2}=g \frac{\mu_{1, j}}{R_{0}} \tanh \left(\frac{\mu_{1, j}}{R_{0}} d\right) .
$$

They are just the natural frequencies of upward cylindrical tank with constant density as proposed in [1]. This coincides with the standard results and verifies the correctness of the solution Eq. (63).

When excitation $\omega \rightarrow N$, the dominator of the first term of Eq.(62) tends to zero. However the numerator of the first term $F_{j}(i \omega)$ contains a term proportional to $N^{2}-\omega^{2}$. The first term is not singular when the excitation is equal to $N$. When external frequency $\omega$ tends to one of the root of $E_{j}(s)=0$, i.e. external forced frequency $\omega$ equals natural frequency $\omega_{j, n}$, both the first and second terms tend to infinity. It can be calculated in the form of

$$
\begin{aligned}
& \lim _{\omega \rightarrow \omega_{j, n}} \frac{F_{j}(i \omega)}{E_{j}(i \omega)\left(N^{2}-\omega^{2}\right)} \frac{\sin (\omega t)}{\omega}+\frac{F_{j}\left(i \omega_{j, n}\right)}{E_{j}^{\prime}\left(i \omega_{j, n}\right)} \frac{2 i}{N^{2}-\omega_{j, n}^{2}} \frac{\sin \left(\omega_{j, n} t\right)}{\omega^{2}-\omega_{j, n}^{2}} \\
& =\left(\frac{2 i E_{j}^{\prime} F_{j}^{\prime} \omega_{j n}-i F_{j} E_{j}^{\prime \prime} \omega_{j n}-E_{j}^{\prime} F_{j}}{2 i E_{j}^{\prime 2}\left(N^{2}-\omega_{j n}^{2}\right) \omega_{j n}^{2}}+\frac{2 F_{j}}{i E_{j}^{\prime}\left(N^{2}-\omega_{j n}^{2}\right)^{2}}\right) \sin \left(\omega_{j n} t\right) \\
& +\frac{F_{j} t \cos \left(\omega_{j n} t\right)}{i E_{j}^{\prime}\left(N^{2}-\omega_{j n}^{2}\right) \omega_{j n}}
\end{aligned}
$$




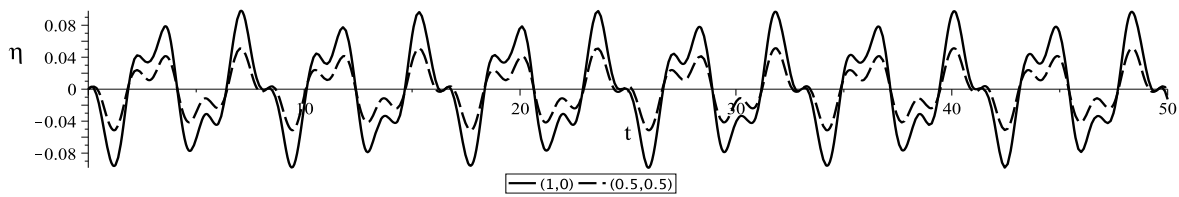

Figure 2: Wave elevation history at two points $(1,0)$ in solid line and $(0.5,0.5)$ in dashed line with $N=0, d=0.6, U_{0}=0.5, \rho_{0}(0)=1, \omega=0.4 \Omega_{1}$ and $R_{0}=1$.

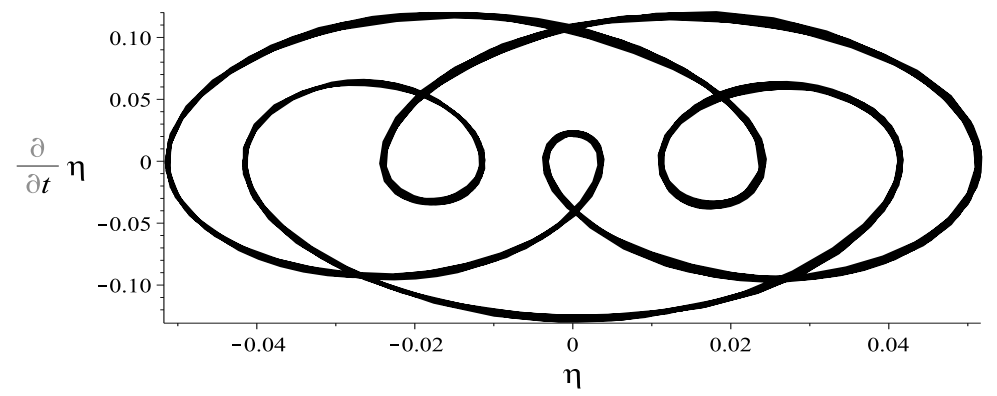

Figure 3: Phase graph of time history of wave elevation at point $(1,0)$ with $N=0, d=$ $0.6, U_{0}=0.5, \rho_{0}(0)=1, \omega=0.4 \Omega_{1}$ and $R_{0}=1$.

by L'hospital rule, where the second term represents resonance, the amplitude become bigger and bigger as time $t$ increases. So the second term of the inverse Laplace transformation tends to infinity as $t \rightarrow \infty$.

Figure 2 shows the wave elevation history at points $(x, y)=(0.5,0.5)$ and $(1,0)$ respectively. The wave elevation means the surface of the liquid. The results are obtained from Eq.(63) with $N=0, d=0.6, R_{0}=1, U_{0}=0.5, \rho_{0}(0)=$ 1 and $\omega=0.4 \Omega_{1}$, where $\Omega_{1}$ is the natural frequency obtained from Eq. (64) for $n=1$. It can be seen that the two figures have similar profiles but the amplitude 125 at point $(1,0)$ is bigger than the amplitude at point $(0.5,0.5)$. This is because the distance from point $(1,0)$ to point $(0,0)$ is longer than distance from $(0.5,0.5)$ to point $(0,0)$. Figure 3 shows the phase graph at point $(0.5,0.5)$ corresponding to Figure 2. The closed phase diagram represents the motion at free surface is periodic.

Figures 4 gives the corresponding resonant behavior of the circular tank sloshing with same parameters in figure 2 except external frequency $\omega$, which is instead of $\Omega_{1}$. They are computed by Eq.(65). The amplitudes increase rapidly when $t$ increases.

Figure 5 shows the wave elevation history of the sloshing circular tank with variable density $N=3, \omega=0.4 \Omega_{1}$ at points $(0.5,0.5)$ and $(1,0)$. The sloshing of stratified liquid gives similar behaviors like constant density liquid. The amplitudes for Brunt-Väisälä frequency $N=3$ are smaller than the amplitudes for uniform density liquid. From the Figure 5 we can see that the amplitude of points $(0.5,0.5)$ and $(1,0)$ are little bigger than the case of constant density. 


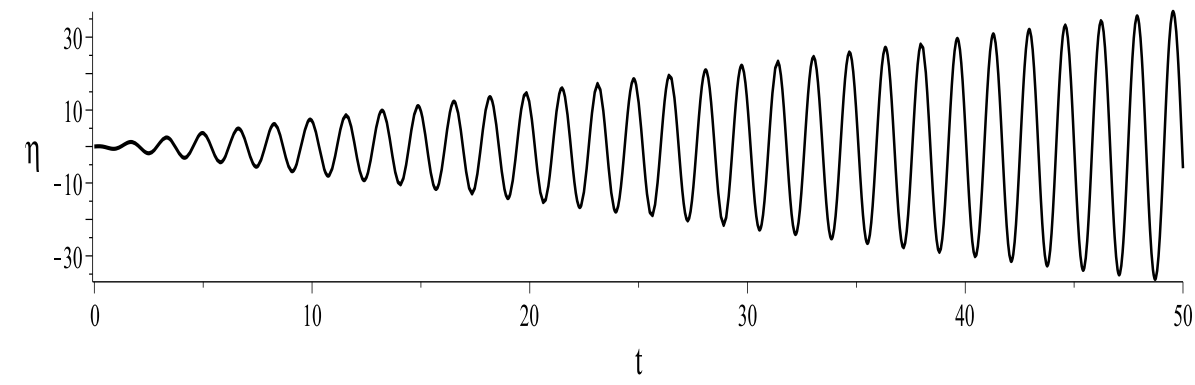

Figure 4: Wave elevation history at point $(0.5,0.5)$ with $N=0, d=0.6, U_{0}=0.5, \rho_{0}(0)=$ $1, \omega=\Omega_{1}$ and $R_{0}=1$.

140 $N=3$ also indicates that the motion is irregular in Figure 6] it is not closed which means the wave motion at point $(0.5,0.5)$ is not periodic.

Figure 7 shows the first four main components of the wave elevation history in Figure 5 at point $(0.5,0.5)$. The first sub-figure denotes the amplitude corof the amplitudes corresponding to the first ten frequencies $\omega_{j, 1}(1 \leq j \leq 10)$ which are solved from Eq.(59), the third sub-figure denotes the amplitudes corresponding to the first ten frequencies $\omega_{j, 2}(1 \leq j \leq 10)$ which are solved from Eq. (61) and the fourth sub-figure denotes the amplitudes corresponding to new

${ }_{150}$ frequencies $\omega_{j, 3}(1 \leq j \leq 10)$ which are solved from Eq.61). The amplitudes of the four sub-figures are 0.054,0.009,0.006 and 0.0006 respectively. we can see that the amplitude of sub-figure (c) is two thirds of the amplitude of sub-figure (b). It means that the amplitudes corresponding to the new natural frequencies $\omega_{j, 2}$ is two thirds of the amplitudes corresponding to natural frequencies $\omega_{j, 1}$. So the amplitudes of each components are given by the coefficients of $\sin \left(\omega_{j, n} t\right)$ in Eq.(63) explicitly. Although the expressions are complex, we can calculate them numerically under given parameters.

The new frequencies $\omega_{j, 3}$ whose action are shown in sub-figure (d) give bigger period and irregular profile. The frequencies $\omega_{j, n}$ decrease while index $n$ increase, then the corresponding periods increase. we also find this in the subfigures (b) (c) and (d) of Figure 7. When $\omega_{j, n}$ tend to zero, the periods tend bigger and bigger from sub-figure (b) to sub-figure (c). But corresponding amplitudes are smaller and smaller.

Figure 8 presents the resonance of the stratified liquid with $N=3$ while external frequency of excitation $\omega$ equals to frequency $\omega_{1,1}$, where $\omega_{1,1} \approx 3.723$ is solved from Eq.(59). It shows similar behavior like constant density liquid in resonant strength. The amplitude increases along with time. Figures 9 shows the resonance with external forced frequency $\omega=\omega_{1,2}$, where $\omega_{1,2} \approx 0.9515$ is 170 the new natural frequency solved from Eq.600 in the interval defined in Eq.61]. The new frequencies caused by continuous stratified flow also could lead to 


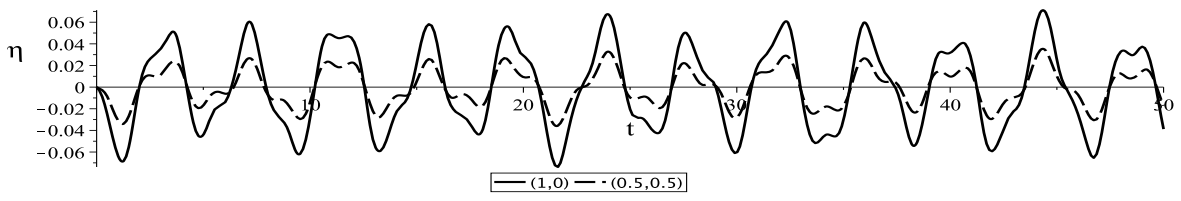

Figure 5: Wave elevation history at point $(1,0)$ in solid line and at point $(0.5,0.5)$ in dashed line with $N=3, d=0.6, \omega=0.4 \Omega_{1}$ and $R_{0}=1$.

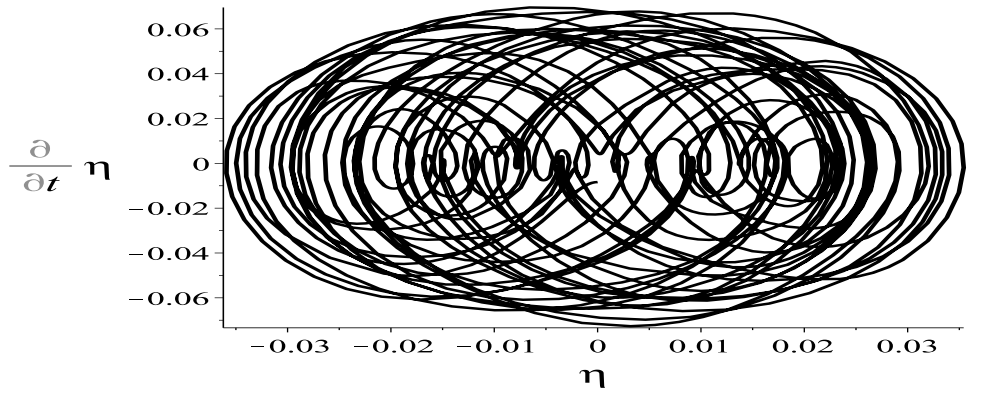

Figure 6: Phase graph of time history of wave elevation at point $(1,0)$ with $N=3, d=$ $0.6, U_{0}=0.5, \rho_{0}(0)=1, \omega=0.4 \Omega_{1}$ and $R_{0}=1$.

resonance when the external frequency equals to the natural frequencies. There are infinite new natural frequencies as implied by Eq. (61). The resonance is more likely to occur compared to the uniform liquid. The resonant motion at some of these new natural frequencies can be significant. But the amplitude of the resonance in Figure 9 is far weaker than the amplitude in Figure 8

Figure 10 presents the first six natural sloshing modes of upwards circular cylindrical tank. They are expressed by Bessel functions $J_{1}\left(\mu_{1, j} \frac{r}{R_{0}}\right) \sin (\theta)$ for $1 \leq j \leq 6$, where $\mu_{1, j}$ are the roots of $J_{1}^{\prime}(r)=0$ in ascending order.

\section{Conclusion}

The sloshing motion is analyzed for the liquid with varying density along the depth. The Laplace equation is not suitable for stratified liquid, and the liquid is no longer irrotional. Despite the two dimensional rectangular tank, the stream function also does not work for three dimensional cylindrical tank.

185 Governing equation and boundary conditions in terms of pressure and density are constructed in order to overcome the problems caused by liquid with variable density. It is found that many new natural frequencies appeared, the resonant behavior is analyzed based on the natural frequencies. We find that these new frequencies have some special characters, they make the motion history of free surface irregular and non-periodic, the amplitudes caused by new frequencies are remarkable, nearly two thirds of the amplitude caused by natural frequencies for $N=3$ and all new frequencies are less than Brunt-Väisäl $\ddot{a}$ frequency $N$. 


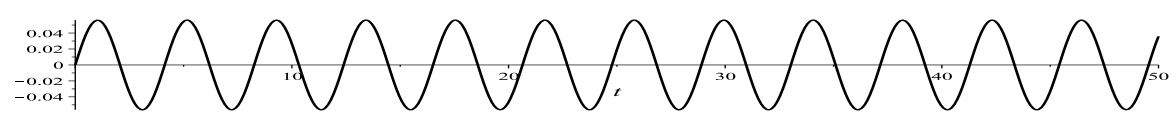

(a) Amplitude component corresponding to external frequency

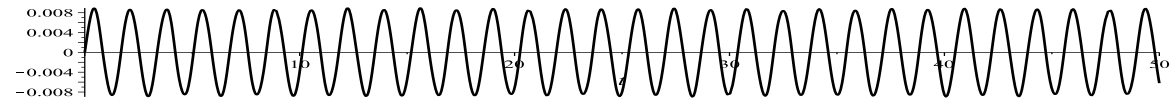

(b) Amplitude component corresponding to the frequencies $\omega_{j, 1}(1 \leq j \leq 10)$ solved from Eq. (59).

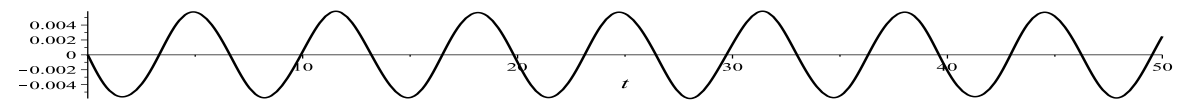

(c) Amplitude component corresponding to the frequencies $\omega_{j, 2}(1 \leq j \leq 10)$ solved from Eq. 61.

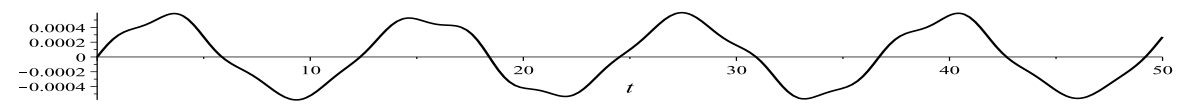

(d) Amplitude component corresponding to the frequencies $\omega_{j, 3}(1 \leq j \leq 10)$ solved from Eq. 61.

Figure 7: Components of Amplitude corresponding to different kinds of frequencies at point $(0.5,0.5)$ with $N=3, d=0.6, U_{0}=0.5, \rho_{0}(0)=1, \omega=0.4 \Omega_{1}$ and $R_{0}=1$.

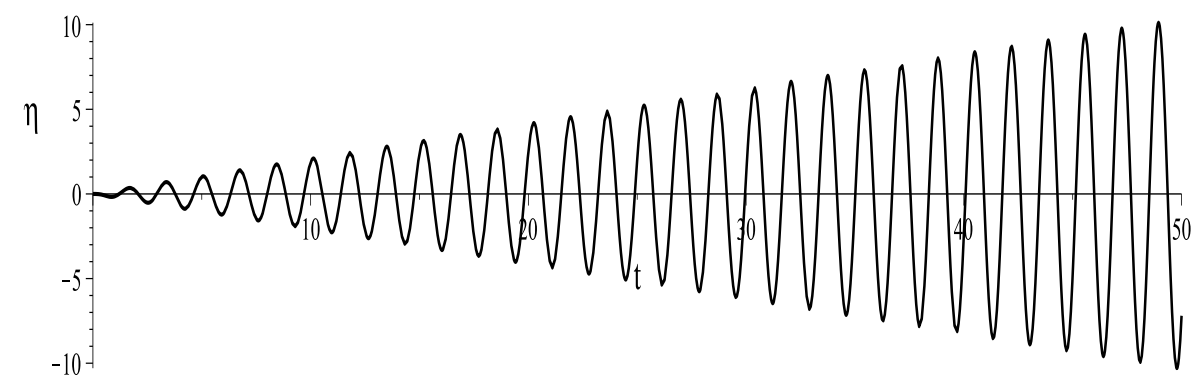

Figure 8: Wave elevation history at point $(0.5,0.5)$ with $N=3, d=0.6, U_{0}=0.5, \rho_{0}(0)=$ $1, \omega=\omega_{1,1}$ and $R_{0}=1$. 


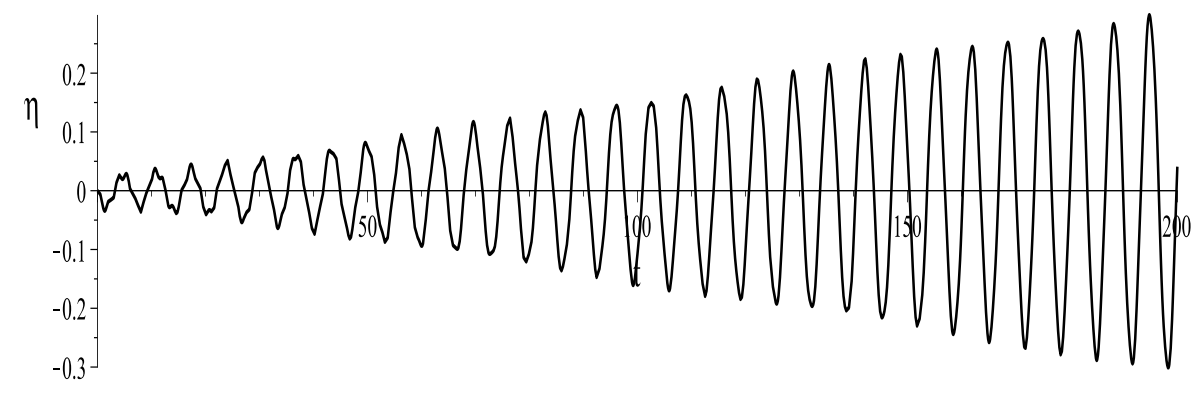

Figure 9: Wave elevation history at point $(0.5,0.5)$ with $N=3, d=0.6, U_{0}=0.5, \rho_{0}(0)=$ $1, \omega=\omega_{1,2}$ and $R_{0}=1$.

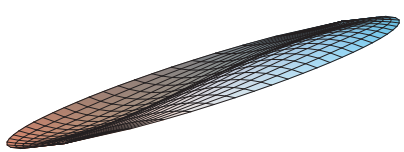

(a) The first sloshing mode $J_{1}\left(\mu_{1,1} \frac{r}{R_{0}}\right) \sin (\theta)$

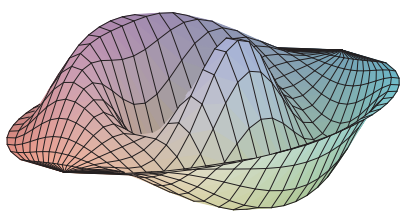

(c) The third sloshing mode $J_{1}\left(\mu_{1,3} \frac{r}{R_{0}}\right) \sin (\theta)$

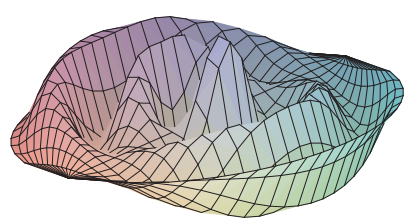

(e) The fifth sloshing mode $J_{1}\left(\mu_{1,5} \frac{r}{R_{0}}\right) \sin (\theta)$

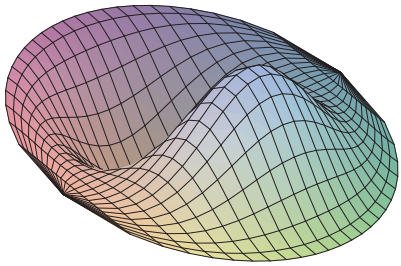

(b) The second sloshing mode $J_{1}\left(\mu_{1,2} \frac{r}{R_{0}}\right) \sin (\theta)$

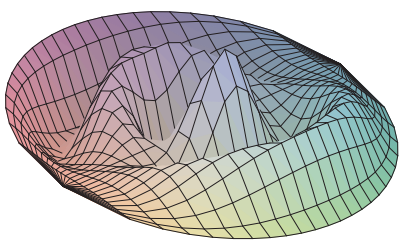

(d) The fourth sloshing mode $J_{1}\left(\mu_{1,4} \frac{r}{R_{0}}\right) \sin (\theta)$

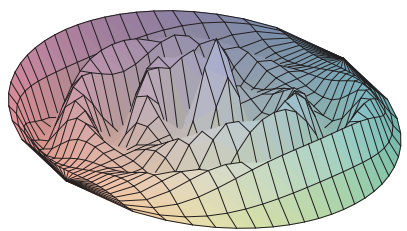

(f) The sixth sloshing mode $J_{1}\left(\mu_{1,6} \frac{r}{R_{0}}\right) \sin (\theta)$

Figure 10: Natural sloshing modes in an upright circular cylindrical tank. 
These characters may be useful for the designing and estimating the safety of the tanks, which contain stratified liquid.

\section{Acknowledgement}

This work is done during visiting Prof. GX Wu in University College London, Thanks to his valuable suggestions. The authors also express thanks anonymous reviewers' advices. Supported by National Science Foundation of China(51579040, 51379033), the Fundamental Research Funds for the Central

200 Universities (DUT2015LK34,DUT2015LK45), and National Key Basic Research Project of China (2013CB036101, 2010CB32700).

\section{References}

[1] Faltinsen OM, Timokha AA. Sloshing. Cambridge University Press, 2009.

[2] J. Lighthill, Waves in Fluids, Cambridge University Press,2001.

[3] C S Yih, Dynamics of Nonhomogeneous fluids, The Macmillan Company, 1965

[4] Batchelor G K. An Introduction to Fluid Dynamics. Cambridge: Cambridge University Press, 1967.

[5] Faltinsen OM. Firoozkoohi R. Timokha AN. Analytical modeling of liquid sloshing in a two-dimensional rectangular tank with a slat screen, Journal of Engineering Mathematics 2011; 70: 93-109.

[6] Tang, Y. Effect of gravitation on dynamic response of tanks containing two liquids. Journal of Engineering Mechanics 1993; 119(3): .

[7] Veletsos AS. Shivakumar P., Hydrodynamic effects in rigid tanks containing layered liquids. Earthquake Engineering Structural Dynamics, 1995; 24(6): 835-860.

[8] Gavrilyuk PI., Lukovsky IA., Timokha AN., Linear and nonlinear sloshing in a circular conical tank, Fluid Dynamics Research 2005; 37: 399-429.

[9] Ardakanin HA, Bridges TJ., Turner MR., Dynamic coupling between horizontal vessel motion and two-layer shallow-water sloshing, Journal of Fluids and Structures, 2015; 59: 432-460.

[10] Sinai Y.L., Fundamental sloshing frequencies of stratified two-fluid systems in closed prismatic tanks.Int. J. of Heat and Fluid Flow, 1985; 6(2): 142144.

[11] Hiroyuki H., Seiichi S., Dynamic behavior of stratified fluids in a rectangular container subject to vertical vibration. Bull JSME. 1985; 28(243): 1910-1917. 
[12] Bandyopadhyay, K. K. "Overview of seismic panel activities." Proc., 3rd DOE natural phenomena hazards mitigation conf., US. Dept. of Energy, Washington, D.C., 1991; 423-429.

[13] Valentine DT., Numerical investigation of two-dimensional sloshing: nonlinear internal waves. J. Offshore Mech. Arct. Eng. 2005; 127(4), 300-305.

[14] Tang, Y. Dynamic response of tank containing two liquids. J. Engrg. Mech. Div., ASCE, 1993; 119(3): 531-548.

[15] Chapman GJD, Porter R. Second-order sloshing over an arbitrary bed. Journal of Fluid Mechanics, 2005; 524: 331-355

[16] Robertson S J, Sherwin S J, Graham J M R. Comparison of wall boundary conditions for numerical viscous fee surface flow simulation. J Fluids Struct, 2004; 19: 525-542

[17] Frandsen J B. Sloshing motion in exited tanks. J Comput Phys B, 2004; 196: $53-87$

[18] Kisheve Z R, Hu C H, Kashiwagi M. Numerical simulation of violent sloshing by a CIP-based method. J Mar Sci Technol, 2006; 11: 111-122

[19] Wu G X, Ma Q W, Eatock Taylor R. Numerical simulation of sloshing waves in a 3D tank based on a finite element method. Appl Ocean Res, 1998; 20: 337-355

[20] Wu GX. Second-order resonance of sloshing in a tank, Ocean Engineering. 2007; 34(17-18): 2345-2349.

[21] Zhang CW. Study on second-order resonance of 3D fully-nonlinear sloshing. International Conference on Maritime Technology 2014, Glasgow, UK.

[22] Faltinsen, O.M., Timokha, A.N. Analytically approximate natural sloshing modes and frequencies in two-dimensional tanks. European Journal of Mechanics B/Fluids, 2014; 47, 176-187

[23] Faltinsen, O.M., Timokha, A.N. Analytically approximate natural sloshing modes for a spherical tank shape. Journal of Fluid Mechanics,2012; 703, 391-401

[24] Faltinsen, O.M., Timokha, A.N. On sloshing modes in a circular tank. Journal of Fluid Mechanics, 2012; 695, 467-477

[25] McIver, P. Sloshing frequencies for cylindrical and spherical containers filled to an arbitrary depth. Journal of Fluid Mechanics, 1989; 201, 243-257

[26] Scolan YM., Some aspects of the eigenfrequency computation in a twodimensional tank filled with two non miscible fluids. 30th International Workshop on Water Waves and Floating Bodies, 2015; Bristol-Bath, UK.

[27] Wu GX. The sloshing of stratified liquid in a two dimensional rectangular tank. Sci China 2011; 54(1): 2-9. 